\title{
Immunohistochemical expression of ubiquitin and telomerase in cervical cancer
}

\author{
Toro de Méndez Morelva • Llombart Bosch Antonio
}

Received: 23 February 2009 /Revised: 7 July 2009 /Accepted: 27 July 2009/Published online: 14 August 2009

(C) Springer-Verlag 2009

\begin{abstract}
Ubiquitin and telomerase immunohistochemical expression patterns in cervical cancer were compared with normal cervical tissue samples. Eighty-one cervical cancer cases and 22 normal exo-endocervical tissue were examined with polyclonal antibody for ubiquitin and 44G12 clone for telomerase using tissue microarrays. The results were interpreted using a semiquantitative scale The average age of patients was 50.67 years. The most frequent histological types were moderately differentiated epidermoid carcinoma (43.5\%), according to the degree of differentiation, and endocervical adenocarcinoma (42.1\%). Immunohistochemical findings were as follows: $98.7 \%$ of cervical cancers showed immunoexpression for ubiquitin and $52.6 \%$ for telomerase. Statistically
\end{abstract}

This study was carried out with support from the AECC (Junta Provincial) and Fundación Instituto Valenciano de Oncología (FIVO), Valencia, Spain.

T. de Méndez Morelva

Exfoliative Cytology,

Faculty of Pharmaceutics and Analytical Biosciences,

University of Los Andes,

Mérida, Venezuela

\section{B. Antonio}

Departament of Pathology, School of Medicine,

University of Valencia,

Valencia, Spain

T. de Méndez Morelva $(\bowtie)$

Facultad de Farmacia y Bioanálisis, Universidad de Los Andes,

Sector Campo de Oro,

5101 Mérida, Venezuela

e-mail: tmorelva@ula.ve

\section{B. Antonio $(\square)$}

Facultad de Medicina y Odontología, Universidad de Valencia,

Av. Blasco Ibáñez, 17,

46010 Valencia, España

e-mail: antonio.llombart@uv.es significant differences were found in tumor immunoreactivity when compared with control tissue $(p<0.0007)$ for both biomarkers. There was no significant difference in biomarker expression at different histological types of tumors, although telomerase was less expressed in endocervical adenocarcinoma. Our findings confirm that abnormal immunoexpression pattern of ubiquitin and telomerase is common in HPVpositive cervical cancer, indicating the existence of an intense degradation of proteins, subsequent cellular immortalization and maintenance of the malignant phenotype.

Keywords Cervical cancer Immunohistochemical expression $\cdot$ Telomerase $\cdot$ Ubiquitin

\section{Introduction}

Cervical cancer and precursor lesions are induced by persistent infection with certain types of high-risk human papillomavirus (HR-HPV) [1, 2]. These viruses are able to activate different molecular mechanisms by inducing the development of a cellular clone with malignant phenotype [3, 4]. Such mechanisms are mediated by proteins used as molecular markers and alteration indicators in cellular control mechanisms. Thus, the study of these complex molecular pathways using different diagnostic techniques such as immunohistochemistry can be conducted. The continual expression of the E6 and E7 HR-HPV oncogenes in high-grade squamous intraepithelial lesions (HSIL) as well as in invasive cancer alter the functionality of a great variety of regulating cellular proteins [5-8]. HPV infection alone is thought to be insufficient for the development of invasive carcinoma.

Telomere length stability is required for the survival of germline human cells and differentiating cells and also for the immortalization of the cellular phenotype derived from 
cancer. This characteristic is achieved through telomerase, a ribonucleoprotein enzyme capable of extending chromosome ends with specific telomeric DNA sequences. This enzyme compensates for the end replication problem and allows cells to proliferate indefinitely $[9,10]$.

Multiple studies have been carried out to evaluate telomerase as a possible HSIL and invasive cervical cancer marker, considering that detection of telomerase activity is useful for cytological screening of cervical lesions using cellular and tissue samples of uterine cervix [6,11-14]. The HR-HPV E6 oncoprotein activates the oncogenic hTERT enzyme, mainly by stimulating the transcription of hTERT gene $[15-17]$, and by immortalizing transformed cervical cells $[6,17]$. Studies have shown that the activity of this enzyme is increased in comparison to normal cervical tissue in cervical cancer and in HSIL HPV 16- and 18-positive samples [17-21].

The rapid removal of enzymes and proteins is essential for the control of cellular growth and metabolism. A rapid intracellular protein degradation pathway is created with the addition of multiple ubiquitin monomers, followed by the recognition and degradation of specific substrate-protein by the $26 \mathrm{~S}$ proteasome [22]. The ubiquitin-proteasome degrades a variety of cellular proteins, which generally have a short half life and include cyclines (D1 and E), cycline-dependent kinase inhibitors (specifically the p27KIP1), tumor-suppressor gene products (p53 and Retinoblastoma protein), transcription factors (E2F), and membrane receptors, among others. Furthermore, those mutated proteins that might also affect cellular homeostasis may be degraded by this pathway $[5,23]$.

Apart from protein degradation, ubiquitin is involved in physiological and pathological cell signaling processes such as gene transcription, DNA repair, and intracellular traffic. This signaling mechanism functions similarly to protein phosphorylation [24]. Substrate proteins that should be degraded are polyubiquitinized during an adenosine triphosphate (ATP)-dependent reaction. Several ubiquitin molecules are added in chains to these proteins. This polyubiquitinized substrate is recognized by the $26 \mathrm{~S}$ proteasome and catalyzes the rupture of the substrate protein until it obtains small peptides of six to 12 aminoacids $[25,26]$.

In many human tumors, the ubiquitin-proteasome protein degradation pathway remains active, with the subsequent rapid degradation of many proteins conveying the lack of control known in cellular proliferation of neoplasias [22]. In the process of cervical carcinogenesis, the integration of HR-HPV in the cellular genome accepts the continuous uncontrolled expression of the HPV E6 and E7 oncogens; both E6/E7 are required for efficient immortalization of their natural host cells. Covalent union of HPV oncoproteins with ubiquitin and other related proteins modifies and induces cellular protein degradation. The E6/E7 oncoproteins are a target of the ubiquitin-proteasome system, and the resultant complex attracts target cellular proteins for their degradation. The ability of E6 and E7 to deactivate the normal function of the main tumor suppressors contributes to their oncogenic potential $[3,7]$.

The HR-HPV 16 and 18 use the cellular proteolytic ubiquitin-proteasome system to target $\mathrm{p} 53$ and $\mathrm{pRb}$ by eliciting rapid degradation of these regulating proteins through the continuous expression of E6 and E7 oncogenes. To facilitate HR-HPV replication and persistence, the infected cells must be irreversibly directed towards the synthesis phase of the cell cycle; as a result, the HR-HPV $\mathrm{E} 7 / \mathrm{pRb}$ complex releases the E2F transcription factor. This interaction leads toward the ubiquitin-proteosome-mediated degradation of $\mathrm{pRb}[27,28]$. The HR-HPV E7 recruits the E3 ubiquitin-ligase enzyme to target the $\mathrm{pRb}$ and cause later degradation. It is also possible that the HR-HPV E7 serves as a direct link between $\mathrm{pRb}$ and the $26 \mathrm{~S}$ proteasome, so that the $\mathrm{pRb}$ may degrade rapidly without previous ubiquitinization $[7,29]$. Thus, the $\mathrm{pRb}$ may be deactivated and rapidly degraded in those cervical epithelial cells infected by HR-HPV.

The specific action of the HR-HPV E6 on p53 is functionally equivalent to $\mathrm{p} 53$ inactivation through mutation, which indicates that the HR-HPV E6/p53 complex represents one of the most important events in cervical carcinogenesis, given the interruption of the control points and apoptosis inhibition. The HR-HPV E6 protein binds to E6-AP, a cellular E3 ubiquitin-ligase, to form a dimer that binds $\mathrm{p} 53$. The complex results in E6AP-mediated ubiquitine and degradation $\mathrm{p} 53$ degradation. As a consequence, the immunohistochemical expression of p53 in neoplastic cervical cells could be negative. This effect could also be affected by the MDM2 protein that induces the ubiquitination and proteasomal degradation of p53 [7]. Degradation of p53 induced by E6-AP is a significant achievement of the HR-HPV, and results in malignant transformation of cervical epithelial cells together with the inactivation and degradation of $\mathrm{pRb}$ [5].

In this study, ubiquitin and telomerase immunohistochemical expression patterns were evaluated in cervical cancer in comparison with normal cervical tissue samples.

\section{Materials and methods}

\section{Materials}

Normal tissue samples (22 cases) and cancer from the uterine cervix were collected from archives of the Pathology Department of the University Hospital of Valencia, Spain. Two groups of cervical cancer samples were 
included; the first with 66 cases diagnosed from 2000 to 2005 and the second with 15 cases of cervical cancer diagnosed between 1969 and 1998. The original hematoxylins of well-preserved cervical cancer cases were reevaluated [21]. Also, 22 cases of normal exocervical and endocervical tissues were included as a control group.

Immunohistochemical study

The tissue microarrays were constructed with distinct tumor areas of squamous cell cervical carcinoma and cervical adenocarcinoma. Tissue microarrays of normal exocervical $(n=22)$ and normal endocervical $(n=22)$ were also prepared taking the exocervical and endocervical sections from each block. Immunohistochemical staining was then carried out. In summary, deparaffinization in xylene and hydration in decreasing alcohol series was performed. Antigen retrieval with citrate buffer $\mathrm{pH} 6.0$ was cooled in the same buffer; subsequently, endogenous peroxidase with $3 \%$ hydrogen peroxide at room temperature for $30 \mathrm{~min}$ was blocked. Similarly, tissue collagen was blocked to avoid nonspecific unions with a $20 \%$ horse serum solution at room temperature for $20 \mathrm{~min}$.

The tissue microarrays were then incubated with primary antibodies for ubiquitin: a polyclonal antibody (DAKO Cytomation, Denmark) diluted at 1:500 and telomerase: 44F 12 clone (Novocastra, UK) for $1 \mathrm{~h}$ at room temperature. The tissue microarrays were then incubated with biotinlabeled secondary antibody contained in the LSAB+ System-horseradish peroxidase (HRP) kit (DAKO Cytomation, Denmark), and liquid diaminobenzidin (DAB)+ Substrate Chromogen System (DAKO Cytomation, Denmark) was used to develop the reaction. All rinses were carried out with phosphate buffered saline (PBS; pH 6) and finally, the tissue microarrays were counterstained with Harris's hematoxylin before mounting. In the specific case of telomerase, the EnVision ${ }^{\mathrm{TM}}$ detection kit, the HRP (DAKO Cytomation, Denmark), and later the chromogene (EnVision ${ }^{\mathrm{TM}}$ ) substrate-DAB solution were used. Immunostaining against the telomerase antigen (diluted at 1:50) was carried out following conventional procedures and contrasting it with Harris's hematoxylin. However, since no satisfactory results were obtained in the case of cervical neoplasia, despite the positive control (lymphoma) showing intense reactivity $(+++)$ for telomerase, a second immunostaining was carried out with diluted antibody 1:20, again using $\left(\mathrm{EnVision}^{\mathrm{TM}}\right)$ and counterstaining with hematoxylin again. Finally, a third immunostaining was carried out, but contrasting with $0.5 \%$ methylium green. Thus, cases with no reactivity (true negatives) were confirmed while those with doubtful reactivity (false negatives) were reclassified.

All rinses were carried out with PBS ( $\mathrm{pH} \mathrm{6).} \mathrm{Negative}$ control included the substitution of a subclass-matched monoclonal antibody that was generated against an irrelevant antigen. Positive controls were made up of neoplastic tissue with known reactivity against the biomarkers under study, from a vesicular tumor, a breast tumor and a lymphoma. These controls were included in each one of the tissue microarrays. Cell counting was carried out including the entire diameter of the tumor disc as the total $(100 \%)$. The positive reaction was represented by the presence of brown precipitation at the membrane, cytoplasm or in both cellular compartments. The percentage of neoplastic or normal cells with reactivity was estimated considering a minimum of 100 cells per tissue. The distribution of the immunoreactive cells was classified according to the following scale: negative $=<5 \%$; positive $=$ $>5 \%$ to $<25 \%$; $++=$ between $25 \%$ and $50 \%$; $+++=>50 \%$. In the case of telomerase, any nuclear staining was interpreted as positive and considered overexpression or abnormal expression. In the case of ubiquitin, cytoplasmic and nuclear immunostaining were considered overexpression or abnormal expression. Moreover, we estimated staining intensity and scored as follows: low, moderate, or high. All tissue microarrays were evaluated first by an observer and later by an experienced pathologist without knowledge of clinical data.

\section{Detection and genotypification of HPV DNA}

Only tumor samples were submitted to HPV evaluation. A paraffin block with no embedded tissue (empty block) was prepared previously. The following recommendations were followed: gloves were used and changed regularly; the microtome was cleaned first with disinfectant, then with xylene, and finally with ethanol; a new knife was used to cut each block and its corresponding empty paraffin block. Five $5 \mu \mathrm{m}$ thick cuts were made and placed in $1.5 \mathrm{ml}$ Eppendorf tubes. We removed the block, cleaned as indicated previously, changed the knife, and cut the corresponding empty paraffin block. The same procedure was used for the subsequent tumor blocks. For isolation of cell DNA, five cuts were submitted for deparaffinization with xylene and washed with absolute ethanol. The pellet was resuspended in $500 \mu \mathrm{l}$ of lysis solution (SDS, $0.5 \%$; Tris-HCl, $10 \mathrm{mM}$; pH 8; NaCl, $0.15 \mathrm{M}$; EDTA, $5 \mathrm{mM}$ ). $25 \mu \mathrm{l}$ of proteinase $\mathrm{K}(0.5 \mathrm{mg} / \mathrm{ml})$ were added to each tube and incubated overnight at $55^{\circ} \mathrm{C}$. DNA was extracted using phenol-chloroform-isoamilic alcohol, and then precipitated by adding one tenth volume of sodium acetate and 2.5 volumes of ethanol. A polymerase chain reaction (PCR) test was carried out before estimating the quality of DNA extracted through amplification of the interferon gene INF primers INF150DR: CTGGGATGCTCTTCGACCTC and INT150DF: TCTTTTCTTTCCCGATAGGT. Samples with a clear band of electrophoresis for INF150 were submitted 
Table 1 Ubiquitin and telomerase expression in case controls and cervical cancer cases

\begin{tabular}{|c|c|c|c|c|c|}
\hline & \multicolumn{2}{|c|}{ Controls $(n(\%))$} & \multicolumn{2}{|c|}{ Cases $(n(\%))$} & \multirow[t]{2}{*}{$p$ value } \\
\hline & Positive & Negative & Positive & Negative & \\
\hline Ubiquitin & $34(76.8)$ & $10(23.2)$ & 79 (98.7) & $1(1.3)$ & 0.0007 \\
\hline Telomerase & $10(22.7)$ & $34(77.3)$ & $41(52.6)$ & $37(47.4)$ & 0.0007 \\
\hline
\end{tabular}

for amplification of the $65 \mathrm{pb}$ segment of the L1 region of HPV DNA using the set of SPF10 primers (INNO-LiPA; Innogenetics Inc., Belgium). The reaction was carried out at a final volume of $50 \mu \mathrm{l}$ containing $2 \mathrm{mM}$ of $\mathrm{MgCl}_{2}$, Triton $\mathrm{X}-100$ at $0.1 \%, 200 \mu \mathrm{M}$ for each dNTPs, $10 \mu \mathrm{l}$ of the mixture of SPF10-biotinylated primers, 1.5 UI of AmpliTaq Gold DNA polymerase (Perkin-Elmer), and $10 \mu \mathrm{l}$ of isolated DNA. The PCR conditions were as follows: initial denaturalization and activation of the polymerase for $9 \mathrm{~min}$ at $94^{\circ} \mathrm{C}$ was followed by 40 cycles of $30 \mathrm{~s}$ at $94^{\circ} \mathrm{C}, 45 \mathrm{~s}$ at $52^{\circ} \mathrm{C}$, and $45 \mathrm{~s}$ at $72^{\circ} \mathrm{C}$, and a final extension for $5 \mathrm{~min}$ at $72^{\circ} \mathrm{C}$. Positive and negative HPV 6 (Innogenetics, Belgium) controls containing only master mix reaction were included. PCR products were analyzed by electrophoresis in $2 \%$ agarose gel stained with ethidium bromide. The samples resulting from positive HPV DNA for SPF10 PCR were submitted for genotypification of HPV using the reverse hybridization band probe assay method (INNOLiPA; Innogenetics Inc., Belgium). The SPF10 PCR products were mixed with a denaturing and hybridized solution with specific probes to identify 24 different HPV types (namely, HPV 6, 11, 16, 18, 31, 33, 35, 39, 40, 42, $43,44,45,51,52,53,54,56,58,59,66,68,70$ and 74$)$.

Statistical analysis

Association between different variables was determined using the Chi-square $\left(X^{2}\right)$ test with the Yates and the Fisher's Exact Probability Test. The SPSS ${ }^{\circledR}$ statistical package was used for the statistical analysis and statistical significance was set at $p<0.05$.

\section{Results}

For this study, the age for each cervical cancer case was obtained from the clinical information originally found at the hospital service (range, 28-79 years; mean, 50.67 years). For the control group the age range was 36-75 years, with a mean age of 51.82 years. A total of 81 cases of cervical cancer were studied: $62(76.5 \%)$ squamous cell cervical carcinomas (SCC) and 19 (23.5\%) cervical adenocarcinomas. In SCC, the moderately differentiated (MD) group tumors $(43.5 \%)$ predominated, followed by welldifferentiated (WD) tumors (21\%). The group of carcinomas with less differentiation was made up of $22.6 \%$ poorly- differentiated (PD) and 12.9\% undifferentiated (UD). The greater portion of adenocarcinomas belonged to the endocervical type (42.1\%), followed by the endometrioid type $(26.3 \%)$; the villoglandular and papillary types reached $15.8 \%$ and $10.5 \%$, respectively; the remaining $5.3 \%$ represented a mucinous type. Forty-four normal cervical tissues (control group: 22 exocervical/22 endocervical) were also included.

Of the 81 cervical tumors, three $(3.7 \%)$ were excluded due to inadequate DNA; the remaining 78 cases $(96.3 \%)$ were all HPV positive, of these, $67(85.9 \%)$ cases showed a single HPV infection and 11 (14.1\%) multiple HPV type infections. The most frequent specific viral type was HPV 16 in $46(58.97 \%)$ cases, followed by HPV 18 in 12 $(15.38 \%)$, HPV 45 in three $(3.84 \%)$, HPV 58 in two (2.56\%), and viral types HPV 31, HPV 66, HPV 68, and HPV $X$ in equal numbers, one of each (1.28\%). The squamous cell carcinomas as well as the adenocarcinomas showed predominantly one type of viral sequence $83.1 \%$ and $94.7 \%$, although they did not reach statistical significance $(p=0.208)$. HPV 16 was the most frequent viral type in SCC $35(71.4 \%)$ as well as in cervical adenocarcinomas ten $(55.6 \%)$, with equal $p$ value 0.248 ; the HPV 18 was detected in $44.4 \%$ of the cervical adenocarcinomas and in $10.2 \%$ of SCC, which were statistically significant $(p<$ $0.001)$. These results are not shown.

Immunoexpression of biomarkers in tumors and controls

Ubiquitin A 98.7\% of cervical cancers showed ubiquitin overexpression; only one case was negative (1.3\%). With respect to case controls, $23.2 \%$ were negative for ubiquitin. We found highly significant differences $(p<0.0007)$ when comparing ubiquitin expression in tumors and case controls; protein degradation was more intense in tumors than in case controls (See Table 1 and Fig. 1).

Fig. 1 Telomerase immunoexpression showing as tiny dots (a and $\mathbf{b}$ normal cervical tissue; $\mathbf{c}$ and $\mathbf{d}$ cervical cancer) within the nuclei and nucleoli (c poorly differentiated carcinoma). The normal exocervical tissue shows telomerase staining at the level of isolated nuclei in the deep strata, while being negative in the endocervix. Ubiquitin (e and $\mathbf{f}$ normal cervical tissue; $\mathbf{g}$ and $\mathbf{h}$ cervical cancer) showed marked expression, both nuclear and cytoplasmic. The normal endocervical epithelium also expresses a low positivity at nuclear and cytoplasmic level. Immunohystochemistry, original magnification: $\mathbf{a}, \mathbf{e} \times 100$ and b, c, d, f, $\mathbf{g}, \mathbf{h} \times 400$ 


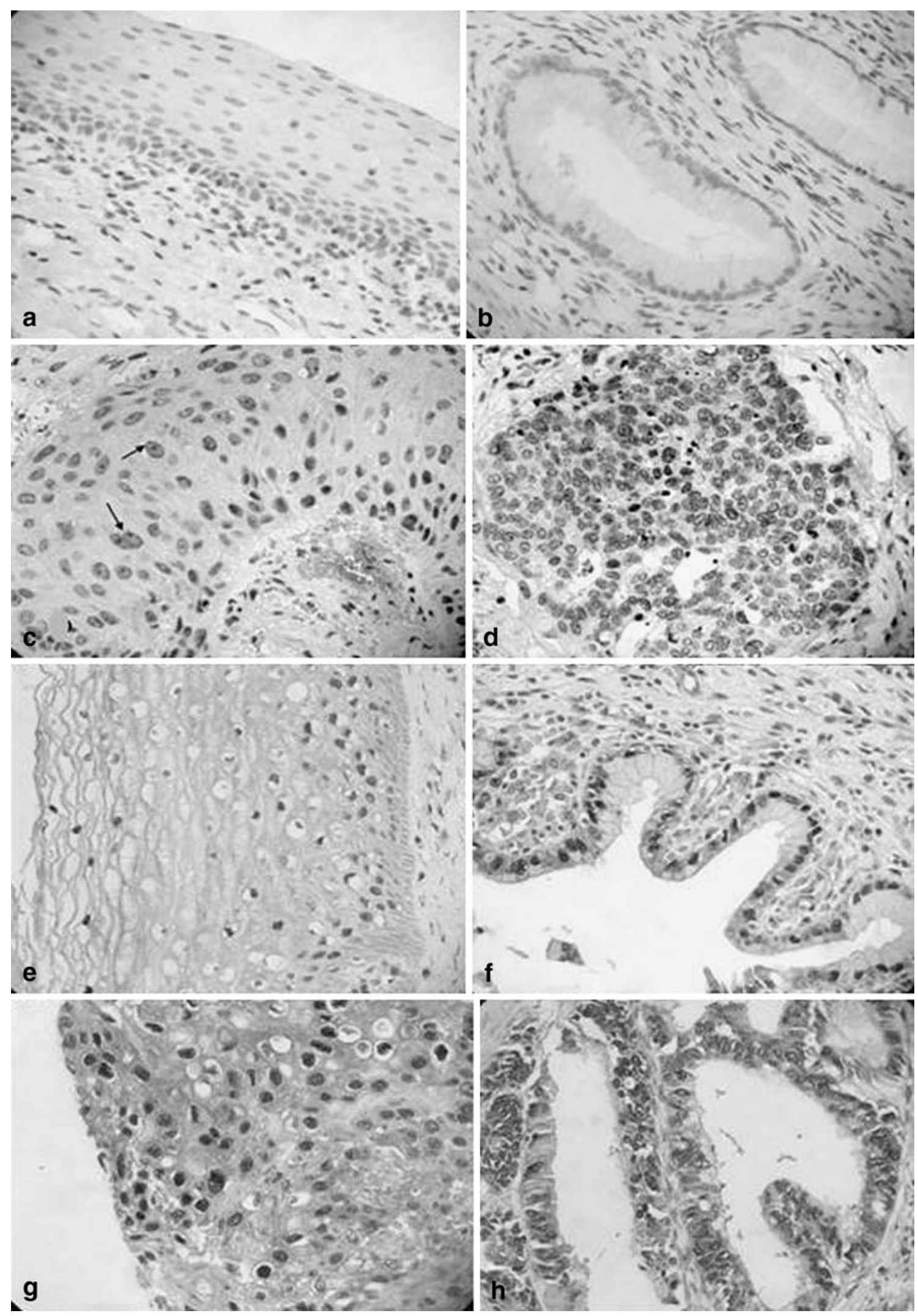


Table 2 Ubiquitin and telomerase expression in histological types of cervical cancer

\begin{tabular}{|c|c|c|c|c|c|}
\hline & \multicolumn{2}{|c|}{ Squamous cells carcinoma $(n(\%))$} & \multicolumn{2}{|c|}{ Adenocarcinoma (n (\%)) } & \multirow[t]{2}{*}{$p$ value } \\
\hline & Positive & Negative & Positive & Negative & \\
\hline Ubiquitin & $60(98.4)$ & $1(1.6)$ & $19(100)$ & - & 0.620 \\
\hline Telomerase & $32(52.5)$ & $29(47.5)$ & $9(52.9)$ & $8(47.1)$ & 0.595 \\
\hline
\end{tabular}

Telomerase Nuclear staining in the lymphocytes was used as a positive internal control in addition to the external positive control that corresponded to a lymphoma. The counterstaining with cytoplasmic $0.5 \%$ methylium green confirmed the negative cases and permitted reclassification with doubtful reactivity. Both internal and external, positive and negative controls showed the expected reactivity. From the cervical cancers, $41(52.6 \%)$ were positive for telomerase, while 37 (47.4\%) did not show reactivity. Case controls were evaluated completely: ten $(22.7 \%)$ positive and 34 (77.3\%) negative. No cytoplasmic staining was observed in any case or in case controls. Telomerase immunoexpression from both tumors and case controls also showed highly significant differences $(p<0.0007)$ given that this enzyme expression was greater in tumors (Table 1).

Immunoexpression of the biomarkers in histological types of cervical tumors

Ubiquitin A 98.4\% of SCCs and 100\% of the adenocarcinomas were positive for ubiquitin, without showing significant differences $(p=0.620)$ (Table 2$)$. Cervical carcinomas showed immunoreactivity for ubiquitin in the nucleus as well as in the cytoplasm. In many cases, nuclear staining was more evident than cytoplasmic, which indicates that protein degradation is constant and occurs in both cellular compartments. Staining intensity was moderate and/or marked. In the stroma, reactivity was also positive (Fig. 1b, d). Ubiquitin was expressed in $100 \%$ of the WD, MD, and UD carcinomas, and $92.9 \%$ of PD $(p=0.879)$. It was also expressed in $100 \%$ of the histological subtypes of the cervical adenocarcinoma without significant differences $(p=0.329)$. The greatest case proportion $14 / 19(73.7 \%)$ was expressed in more than $50 \%$ of malignant cells. These results are summarized in Table 3 .

Telomerase Regarding the distribution of cervical carcinomas with or without telomerase expression, we observed that both types of tumors were positive, showing similar percentages $(52.5 \%$ for SCCs and $52.9 \%$ for adenocarcinomas) and with no statistically significant differences ( $p=$ 0.595 ) (Table 2). The immunoreactivity was only nuclear and nucleolar. The staining pattern was characterized by a very fine granular homogenous appearance and a pale sharp-edge aspect. The nucleus of both malignant cells and stromal lymphocytes showed reactivity, this being more intense. The nucleoli turned greyish-brown and became highly noticeable within the nucleus. In general, staining was less intense. In Table 3, telomerase immunoexpression in the histological types of cervical cancer is shown in detail. Reactivity resulted in similar percentages between the various degrees of SCCs differentiation, being higher at UD (62.5\%), followed by the MD (55\%), and the same proportion in the WD and PD (46.2\%), with no significant differences $(p=0.667)$. Cervical adenocarcinoma showed telomerase expression with statistically significant differences between the different histological types $(p<0.028)$ : in $100 \%$ of the villoglandular type and in the unique mucinous case, followed by the endometrioid and papillary histological subtypes in equal proportions $(50 \%)$. The endocervical adenocarcinoma showed a small proportion of reactive cells $(28.6 \%)$. No correlation between HPV presence, and ubiquitin $(p=0.305)$ and telomerase $(p=0.656)$ immunoexpression was observed.

Immunoexpression of biomarkers in normal cervical tissues

Ubiquitin The nucleus at the deep layer of exocervical tissue together with some intermediate cells were stained moderately-to-slightly. Pale cytoplasmic staining was also observed. Stroma staining was more evident in the vascular endothelium (Fig. 1a). In normal endocervical epithelium, staining was nuclear, intense, and focally cytoplasmic. Stroma cells nuclei and cytoplasms were stained (Fig. 1c). We included a special positive case control to compare ubiquitin immunostaining which corresponded to a nongestational endometrial tissue. This resulted in mainly cytoplasmic staining and some highly intense nuclei.

Telomerase Occasional staining in cellular nuclei was observed (less than $10 \%$ of cells) in deep layer exocervical tissue. There was no nuclear staining in intermediate and superficial layers. Stroma staining was sporadic. Some endocervical cells and stromal nuclei were stained.

\section{Discussion}

In this study, highly significant differences in ubiquitin detection between study cases and case controls have been observed. In cervical lesions associated with HR-HPV, 
Table 3 Immunoreactivity of malignant cells in the squamous cell carcinomas according to degree of differentiation and in the histological subtypes of cervical adenocarcinoma

\begin{tabular}{|c|c|c|c|c|c|c|c|}
\hline Cervical cancer & & Scale & Ubiquitin & $p$ value & Scale & Telomerase & $p$ value \\
\hline \multirow[t]{13}{*}{ Squamous cell carcinoma } & Grade of differentiation & & & 0.879 & & & 0.667 \\
\hline & WD & $<5 \%$ & - & & $<5 \%(-)$ & 53.8 & \\
\hline & & $5-50 \%$ & 23.1 & & $>5 \%(+)$ & 46.2 & \\
\hline & & $>50 \%$ & 76.9 & & & & \\
\hline & MD & $<5 \%$ & - & & $<5 \%(-)$ & 44.4 & \\
\hline & & $5-50 \%$ & 38.5 & & $>5 \%(+)$ & 55.6 & \\
\hline & & $>50 \%$ & 61.5 & & & & \\
\hline & PD & $<5 \%$ & 7.1 & & $<5 \%(-)$ & 53.8 & \\
\hline & & $5-50 \%$ & 28.6 & & $>5 \%(+)$ & 46.2 & \\
\hline & & $>50 \%$ & 64.3 & & & & \\
\hline & UD & $<5 \%$ & - & & $<5 \%(-)$ & 37.5 & \\
\hline & & $5-50 \%$ & 12.5 & & $>5 \%(+)$ & 62.5 & \\
\hline & & $>50 \%$ & 87.5 & & & & \\
\hline \multirow[t]{11}{*}{ Cervical adenocarcinoma } & Histological subtypes & & & 0.329 & & & 0.028 \\
\hline & Endocervical & $25-50 \%$ & 25 & & $<5 \%(-)$ & 71.4 & \\
\hline & & $>50 \%$ & 75 & & $>5 \%(+)$ & 28.6 & \\
\hline & Mucinous & $25-50 \%$ & - & & $<5 \%(-)$ & - & \\
\hline & & $>50 \%$ & 100 & & $>5 \%(+)$ & 100 & \\
\hline & Endometrioid & $25-50 \%$ & 20 & & $<5 \%(-)$ & 50 & \\
\hline & & $>50 \%$ & 80 & & $>5 \%(+)$ & 50 & \\
\hline & Papillary & $25-50 \%$ & - & & $<5 \%(-)$ & 50 & \\
\hline & & $>50 \%$ & 100 & & $>5 \%(+)$ & 50 & \\
\hline & Villoglandular & $25-50 \%$ & 66.7 & & $<5 \%(-)$ & - & \\
\hline & & $>50 \%$ & 33.7 & & $>5 \%(+)$ & 100 & \\
\hline
\end{tabular}

$W D$ well differentiated, $M D$ moderately differentiated, $P D$ poorly differentiated, $U D$ undifferentiated

these viruses are considered a vital mechanism for the degradation of the main regulating cell proteins, through the elimination of the check points that protect the cell from alterations of its internal homeostasis, such as the tumor suppressing protein $\mathrm{p} 53[7,30,31]$ and the Retinoblastoma protein $[7,28]$. Similarly, HR-HPV oncoproteins use the ubiquitin-proteosome system to target many other cell regulatory proteins for degradation [8]. In consequence, not only cell proteins are functionally inactive, but their expression levels tend to be lower due to intense degradation [7]. In this study, we have found a high expression of ubiquitin in cervical carcinoma, which indicates an intense protein degradation, regardless of the degree of differentiation in squamous cell carcinoma, although more intense in the poorly differentiated cases, and quite similar in the various subtypes of adenocarcinomas.

Immortalization of telomerase enzyme activation has been detected in malignant neoplasias, considering its expression as an essential step towards malignant transformation of human tissue $[6,32,33]$. This capacity allows unlimited multiplication of malignant cells. Multiple studies have been carried out to evaluate telomerase as a possible marker of HSIL and cancer and have found it useful as a complement to cytological diagnosis, especially in patients with cytological abnormalities associated with HR-HPV [6, 11-14]. These studies used the Telomeric Repeat Amplification Protocol TRAP method, which permitted the determination of enzymatic activity by amplifying PCR, in which up to $100 \%$ of this enzyme expression has been reported $[19,21,33,34]$. In the present study, $52.6 \%$ of cervical cancer cases showed telomerase immune expression. These differences are possibly due to a higher sensitivity of the method used for capturing and amplifying the reduced quantity of telomerase synthesized in malignant and premalignant cervical cells and to the criteria used for evaluating enzymatic expression. Despite the advantages offered by molecular techniques, it is also possible to estimate telomerase expression in human cervical samples through immunohistochemistry. This technique has the advantage of allowing accurate observation of localized protein expression in premalignant and malignant cells in cervical tissue samples [35]. 
Efficiency of telomerase immunodetection depends on the conditions related to an adequate dilution of primary antibodies and on the use of highly sensitive antigenantibody reaction detection systems. In addition, for doubtful cases, due to the observation of a fine and homogeneous sharp-edged immunoreactive pattern, it is necessary to use a cytoplasmic counterstaining that permits better nuclear observation and differentiation staining. It is possible that, the percentage of this enzyme expression may be higher in our tumor series because it may influence a dilution of inappropriate anti-hTERT antibodies. This could be proved in future research studies, where other forms of antigenic recovery $[35,36]$ might be considered.

In this study, $53.8 \%$ of the WD carcinomas were negative for telomerase, while UD showed positively in $62.5 \%$ of the cases. It is possible that tumor heterogeneity may influence the enzymatic analysis. As Jorboe et al. [6] explained, the telomerase activity could be related to the degree of tumor differentiation. In fact, well-differentiated tumors have less enzymatic expression, as shown in our results.

With respect to the endocervical adenocarcinomas, $71.4 \%$ were negative telomerase ( $<5 \%$ of reactive cells). These tumor cells may be found in apoptosis [35], telomerase inhibiting factors may exist when blocking their activation, and the enzyme may present qualitative modifications (hypophosphorylation) [36, 37]. In addition, hTERT variants not transported to the cytoplasm nucleus could be detected for their activation [35]. Finally, perhaps the cell may synthesize at minimum or nondetectable enzymatic levels, together with the development of other mechanisms that may keep the malignant phenotype, among other causes [38]. Future research could give answers to these hypotheses.

Normal cervical epithelium showed less immunoreactivity against the studied biomarkers when compared with the neoplastic tissue, which revealed abnormal expression in tumors with statistically significant differences. The biomarker immunoexpression in neoplastic tissue compared with the normal cervical tissue confirmed that in malignant cervical neoplasia, a diversity of interactions and altered molecular mechanisms induce cycle deregulation and uncontrolled cell proliferation. These alterations are apparently produced by HR-HPV E6/E7 oncoproteins associated with telomerase activation in cells with genomic instability and activated myc gene. The formation of E6-myc complex stimulates the expression of the hTERT gene by activating the promoter of this gene [39, 40]. HR-HPV E6 expression is relevant for the process of cell immortalization and suggests that other cell genes (myc) could be regulated by this viral protein in favor of cellular progression and immortalization [41].
The present findings confirm that an abnormal immunoexpression pattern of ubiquitin and telomerase, determined by immunodetection, is common in HPV-positive cervical cancer in comparison with normal cervix. This may be related to the existence of an intense protein degradation, with a consequent cellular immortalization which leads to the maintenance of a malignant phenotype.

Acknowledgments The authors thank Alejo Sempere and Laura Martínez from the Department of Pathology, School of Medicine, University of Valencia, for their invaluable support and training immunohistochemical technicians.

Conflict of interest statement We declare that we have no conflict of interest.

\section{References}

1. Muñoz N, Bosch FX, de Sanjosé S et al (2003) Epidemiologic classification of human papillomavirus types associated with cervical cancer. N Engl J Med 348:518-527

2. Moscicki A-B, Schiffman M, Kjaer S et al (2006) Updating the natural history of HPV and anogenital cancer. Vaccine 24S3:S3/ $42-\mathrm{S} 3 / 51$

3. zur Hausen H (2000) Papillomaviruses causing cancer: evasion from host-cell control in early events in carcinogenesis. J Natl Cancer Inst 92:690-698

4. von Knebel-Doeberitz M (2001) Aspects of molecular pathogenesis of cervical cancer in establishing new tumor markers for early detection and diagnosis. Zentralbl Gynakol 123:186-191

5. Scheffner M (1998) Ubicuitin, E6-AP, and their role in p53 inactivation. Pharmacol Ther 78:129-139

6. Jarboe EA, Liaw K-L, Thompson LC et al (2002) Analysis of telomerase as a diagnostic biomarker of cervical dysplasia and carcinoma. Oncogene 21:664-673

7. Scheffner M, Whitaker NJ (2003) Human papillomavirus-induced carcinogenesis and the ubicutin-proteosome system. Sem Cancer Biol 13:59-67

8. Kim YT, Zhao M (2005) Aberrant cell cycle regulation in cervical cancer. Yonsei Medical J 46:597-613

9. Hanahan D, Weinberg R (2000) The hallmark of cancer. Cell 100:57-70

10. Hiyama E, Hiyama K (2007) Telomere and telomerase in stem cell. British J Cancer 96:1020-1024

11. Reesink-Peters N, Helder MN, Wisman GBA et al (2003) Detection of telomerase, its components, and human papillomavirus in cervical scrapings as a tool for triage in women with cervical dysplasia. J Clin Pathol 56:31-35

12. Braviccini S, Sanchini MA, Amadori A et al (2005) Potencial of telomerase expresión and activity in cervical specimens as a diagnostic tool. J Clin Pathol 58:911-914

13. Jarboe EA, Thompson LC, Heinz D et al (2004) Telomerase and human papillomavirus as diagnosis adjuncts for cervical dysplasia and carcinoma. Hum Pathol 35:396-402

14. Pinto-Tang CT, Premoli G (2005) Detection of telomerase activity in cervical lesions by non-radioactive telomeric repeat amplification protocol (TRAP). Invest Clin 46:255-263

15. Gewin L, Galloway DA (2001) E box-dependent activation of telomerase by human papillomavirus type 16 E6 does not require induction of c-myc. J Virol 75:7198-7201 
16. Oh ST, Kyo S, Laimins LA (2001) Telomerase activation by human papillomavirus type 16 E6 protein: induction of human telomerase reverse transcrptase expression through $M y c$ and GCrich Sp1 binding sites. J Virol 75:5559-5566

17. Branca M, Giorgi C, Ciotto M et al (2006) Upregulation of telomerase (hTERT) is related to the grade of cervical intraepithelial neoplasia, but is not an independent predictor of highrisk human papilomavirus, virus persistence, or disease outcome in cervical cancer. Diagn Cytopathol 34:739-748

18. Takakura M, Saturo K, Taro K et al (1998) Expression of human telomerase subunits and correlation with telomerase activity in cervical cancer. Cancer Res 58:1558-1561

19. Wang S-Z, Sun J-H, Zhang W et al (2004) Telomerase activity in cervical intraepithelial neoplasia. Chinese Med J 117:202-206

20. Anderson S, Shera K, Ihle J et al (1997) Telomerase activation in cervical cancer. Am J Pathol 151:25-31

21. Yashima K, Ashfaq R, Nowak J et al (1998) Telomerase activity and expression of its RNA component in cervical lesions. Cancer 82:1319-1327

22. Pagano M (1997) Cell cycle regulation by the ubicutin pathway. FASEB J 11:1067-1075

23. Rolfe M, Chiu I, Pagano M (1997) The ubicutin-mediated proteolytic pathway as a therapeutic area. J Mol Med 75:5-17

24. Haglund K, Dikic I (2005) Ubicuitylation and cell signaling. EMBO J 24:3353-3359

25. Huibregtse JM, Scheffner M, Beaudenon S et al (1995) A family of proteins structurally and functionally related to the E6-AP ubiquitin-protein ligase. Proc Natl Acad Sci USA 92:2563-2567

26. Mitch WE, Goldberg AL (1996) Mechanisms of muscle wastingthe role of the ubiquitin-proteosome pathway. New Engl J Med 335:1897-1905

27. Boyer SN, Wazer DE, Band V (1996) E7 protein of human papilloma virus-16 induces degradation of retinoblastoma protein through the ubicutin-proteosome pathway. Cancer Res 56:4620-4624

28. González SL, Stremlau M, He X et al (2001) Degradation of the retinoblastoma tumor suppressor by the human papillomavirus type 16 E7 oncoprotein is important for functional inactivation and is separable from proteosomal degradation of E7. J Virol 75:7583-7591

29. Berezutskaya E, Bagchi S (1997) The human papillomavirus E7 oncoprotein functionally interacts with the S4 subunit of the $26 \mathrm{~S}$ proteosome. J Biol Chem 272:30135-30140
30. Kao WH, Beaudenon SL, Talis AL et al (2000) Human papillomavirus type 16 E6 induces self-ubicuitination of the E6AP ubiquitin-protein ligase. J Virol 74:6408-6417

31. Hengstermann A, Linares LK, Ciechanover A et al (2001) Complete switch from Mdm2 to human papillomavirus E6mediated degradation of $\mathrm{p} 53$ in cervical cancer cells. Proc Natl Acad Sci USA 98:1218-1223

32. Shay JW, Bacchetti S (1997) A survey of telomerase activity in human cancer. Eur J Cancer 33:787-791

33. Yokoyama Y, Takahashi Y, Shinohara A et al (1998) Telomerase activity in the female reproductive tract and neoplasms. Gynecol Oncol 68:145-149

34. Shroyer KR, Thompson LC, Enomoto T et al (1998) Telomerase expression in normal epithelium, reactive atypia, squamous dysplasia, and squamous cell carcinoma of the uterine cervix. Am J Clin Pathol 109:153-162

35. Yan P, Benhattar J, Seelentag W et al (2004) Immunohistochemical localization of hTERT protein in human tissues. Histochem Cell Biol 121:391-397

36. Kyo S, Masutomi K, Maida Y et al (2003) Significance of immunological detection of human telomerase reverse transcriptase. Re-evaluation of expression and localization of human telomerase reverse transcriptase. Am J Pathol 163:859-867

37. Liu K, Hodes RJ, Weng NP (2001) Cutting edge: telomerase activation in human $\mathrm{T}$ lymphocytes does not require increase in telomerase reverse transcriptase (hTERT) protein but is associated with hTERT phosphorylation and nuclear translocation. J Immunol 166:4826-4830

38. Kakihana M, Yahata N, Hirano T et al (2002) Telomerase activity during carcinogenesis in the bronchus. Oncol Rep 9:4349

39. Veldman T, Horikawa I, Barrett JC et al (2001) Transcriptional activation of the telomerase $h T E R T$ gene by human papillomavirus type 16 E6 oncoprotein. J Virol 75:4467-4472

40. Fehrmann F, Laimins LA (2003) Human papillomaviruses: targeting differentiation epithelial cells malignant transformation. Oncogene 22:5201-5207

41. Veldman T, Liu X, Yuan H et al (2003) Human papillomavirus E6 and $M y c$ proteins associate in vivo and bind to and cooperatively activate the telomerase reverse transcriptase promoter. PNAS 100:8211-8216 